\title{
UNILATERAL INTERCORDAL NEURAL COMMUNICATION COEXISTENT WITH VARIANT BRANCHING PATTERN OF POSTERIOR CORD OF BRACHIAL PLEXUS
}

\author{
Renu Baliyan, Vandana Mehta, Jyoti Arora, Ashish Kr. Nayyar, R. K. Suri, Gaytri Rath
}

\author{
Department of Anatomy, Vardhman Mahavir Medical College, New Delhi, India
}

\begin{abstract}
Summary: Variant branching pattern of the cords of brachial plexus coupled with erroneous communications has been an area of concern for surgeons opting to explore this region. Anaesthetic blocks and surgical approaches are the highlights of these interventions, where a keen familiarization of the anatomy of this region is mandatory. The present case description reports a unilateral variant branching pattern of the posterior cord coexistent with a neural communication between lateral and medial cords in an adult male cadaver. This intercordal neural communication between lateral and medial cords was oriented obliquely and measured $2.2 \mathrm{~cm}$ in length. Furthermore, the posterior cord revealed a variant branching pattern. It branched into three upper subscapular nerves and a common trunk for the thoracodorsal and lower subscapular nerves. The lowest of the three upper subscapular nerves gave a communicating twig to the thoracodorsal nerve. Inspite of uncountable reports on variations of brachial plexus, descriptions regarding anomalous branching patterns hold enormous clinical significance for the radiologists, anesthetists and surgeons, besides being of academic interest for the anatomists.
\end{abstract}

Key words: Posterior cord; Medial cord; Lateral cord; Neural communication

\section{Introduction}

The hallmark of the axillary region is the brachial plexus, which is a plexiform arrangement of the anterior primary rami of the lowest 4 cervical and first thoracic spinal nerves. It is disposed in two parts, the supraclavicular and infraclavicular. The supraclavicular part consists of the roots and the trunks, whereas the infraclavicular part has the cords placed in relation to the axillary artery. The plexus is intimately related to neurovascular structures both in the cervical and the axillary regions. There have been innumerable descriptions of brachial plexus variations, particularly pertaining to the plexus as a whole. The nerves of brachial plexus are oriented in two planes: anterior and posterior. The anterior plane consists of the branches of the medial and lateral cords. It has been stated earlier that connections and variations are frequently found in the anterior plane $(1,14)$.

An astonishing part of the current case report is the presence of anomalies in both planes, anterior and posterior. We are in total agreement with a prior statement that the variations of cords and the individual branches are rarer and uncommonly reported in anatomical literature (11).

The traditional view of the median nerve being formed by the medial and lateral roots and positioned in front of the axillary artery may not always be the case and may show deviations from the usual norm (10).

The present investigation strives to report a unilateral occurrence of a variant branching pattern of the posterior cord along with an intercordal neural communication between medial and lateral cords of brachial plexus.

\section{Case report}

We detected an unusual organization and branching pattern of the left brachial plexus during the course of a routine preclinical educational dissection of an adult male cadaver. A neural communication between the lateral and medial cords was observed. This intercordal communication crossed superficially to the axillary artery in an oblique manner and measured $2.2 \mathrm{~cm}$ in length. The medial cutaneous nerve of the forearm originated from the proximal part of the medial cord and then traversed deep into the neural communication between the lateral and medial cords. The thoracoacromial branch of the axillary artery was seen crossing superficially to the lateral cord of the brachial plexus. The median nerve was formed as usual by the union of medial and lateral roots arising from respective cords of the brachial plexus. The ulnar nerve and medial root of the median nerve were found originating from the medial cord, distal to the neural communication between the lateral and medial cords. The posterior cord showed a divergence from the usual branching pattern. It branched into three upper subscapular nerves. Distally, a common trunk originating from posterior cord was found to divide into the thoracodorsal and lower subscapular nerves. The lowest of the three upper subscapular nerves gave a communicating twig to the thoracodorsal nerve. No 
other neural variations were noted. In addition, the vessels and musculature revealed no departure from the normal.

\section{Discussion}

Volumes of anatomical literature have been written on the variations of the brachial plexus. A thorough survey of the archives revealed that communication between the median and musculocutaneous nerves are the most frequent anomalies reported. However, variations pertaining to the roots and cords are relatively uncommon. Anomalies in the formation of brachial plexus cords have been reported with varying frequencies of $6.2 \%(5), 5.8 \%(11), 0.57 \%(18)$ and $3 \%(16)$.

The presence of an intercordal neural communication between the medial and lateral cords of the brachial plexus coexistent with a variant branching pattern of the posterior cord contribute to the exceptionality of the current case report, although earlier studies have described the same $(4,7,11)$.

A credible embryological elucidation has been offered for neural variations of the upper limb. It is already an estab-

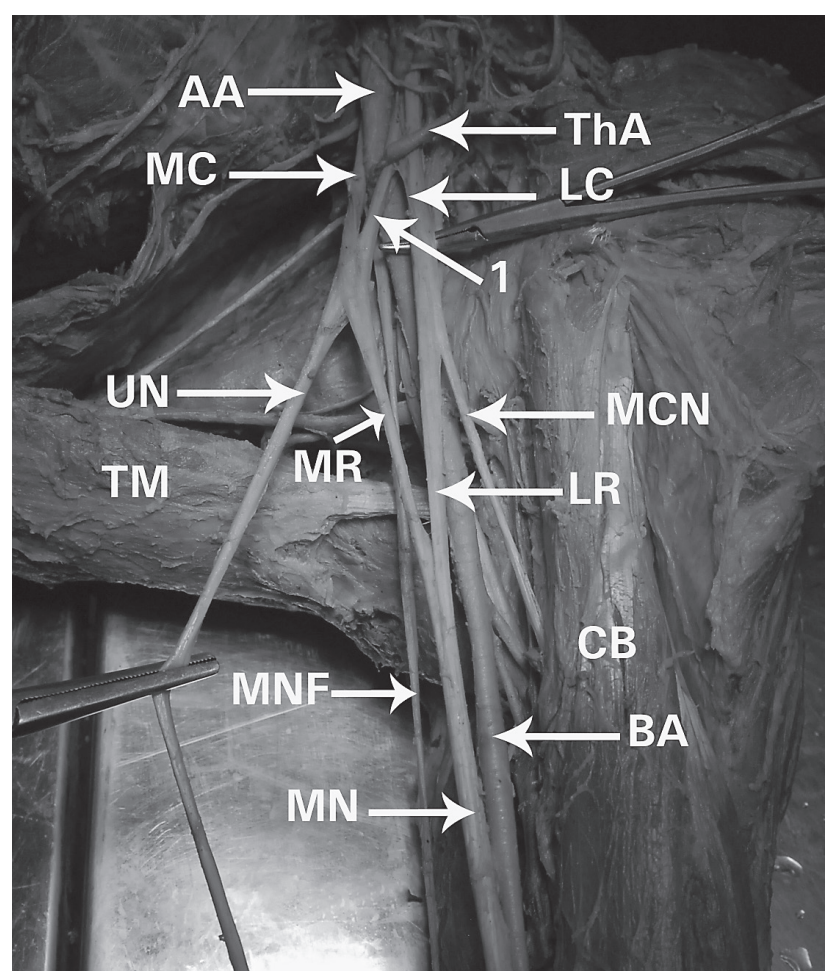

Fig. 1: Neural communication between lateral and medial cord. LC - lateral cord, MC - medial cord, LR - lateral root, $\mathrm{MR}$ - medial root, $\mathrm{MN}$ - median nerve, UN - ulnar nerve, AA - axillary artery, TM - teres major muscle, $\mathrm{CB}$ - coracobrachialis muscle, MNF - medial cutaneous nerve of forearm, MCN - musculocutaneous nerve, BA brachial artery, ThA - thoracoacromial artery, 1 - neural communication lished fact that as soon as the upper limb buds are formed, the ventral primary rami of the spinal nerves penetrate the mesenchyme of the bud. Each ventral ramus then divides into ventral and dorsal branches which later unite to form the known peripheral nerves for the flexors and extensors of the upper extremity, respectively. The contact between the nerves and the differentiating mesodermal condensations is mandatory for accomplishing the functional differentiation (12). Lack of coordination between these processes may lead to inter-neural deviations from the usual branching pattern $(10,17)$.

The intercordal neural communication between the medial and lateral cords of the brachial plexus encountered in the current study is clinically significant. These supernumerary communications are susceptible to damage during radical neck dissections and surgical interventions in the upper arm and axilla (16). It is an established fact that the knowledge of neural connections has immense relevance in diagnostic neurophysiology (2).

The medial cutaneous nerve of the forearm traversing deep into the neural communication between the lateral and medial cords is an interesting observation in the present investigation. The authors speculate that this unusual relationship may possibly misguide the anesthetist performing a brachial plexus block.

In the present study it is also pertinent to note the site of origin of the ulnar nerve and the medial root of the median nerve from the medial cord of the brachial plexus distal to the neural communication between the lateral and medial cords. This observation lends credence to the possible con-

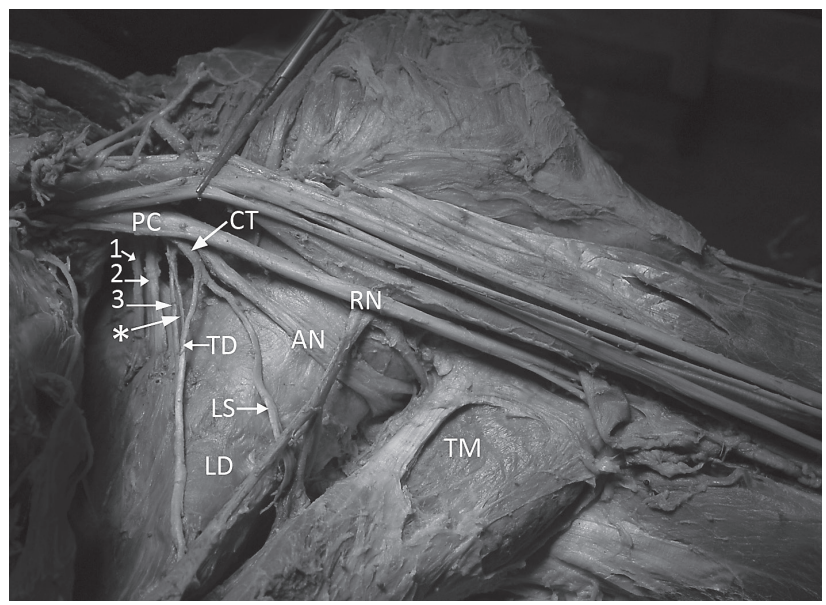

Fig. 2: Variation in the branching pattern of the posterior cord of left side. PC - posterior cord, 1 - upper subscapular nerve, 2 - upper subscapular nerve, 3 - upper subscapular nerve, CT - common trunk, TD - thoracodorsal nerve, LS - lower subscapular nerve, LD - latissmus dorsi muscle, * - communicating twig of 3rd upper subscapular nerve to the common trunk of thoraco-dorsal and lower subscapular nerves, TM - teres major muscle, $\mathrm{RN}$ - radial nerve, $\mathrm{AN}$ - axillary nerve 
tribution of lateral cord as well for the origin of these nerves.

The upper subscapular nerve revealed wide ranging variations in its origin (15). Among the commonly found origins of upper subscapular nerves are the suprascapular and eight cervical spinal nerves (3).

The present study displayed the presence of three upper subscapular nerves. Furthermore, the communication between the lowest upper subscapular nerve and thoracodorsal nerve is also an interesting finding in the current study. This neural communication may possibly serve to maintain innervation for latissimus dorsi muscle in the event of injury to thoracodorsal nerve.

We as anatomists also submit that the communication between the thoracodorsal and subscapular nerves encountered in the present study would presumably facilitate coordination of the posterior axillary wall musculature.

Surgical literature has reported the usage of left latissimus dorsi muscle in repairing failing left ventricles (9). The left latissimus dorsi muscle is ideal for this purpose because

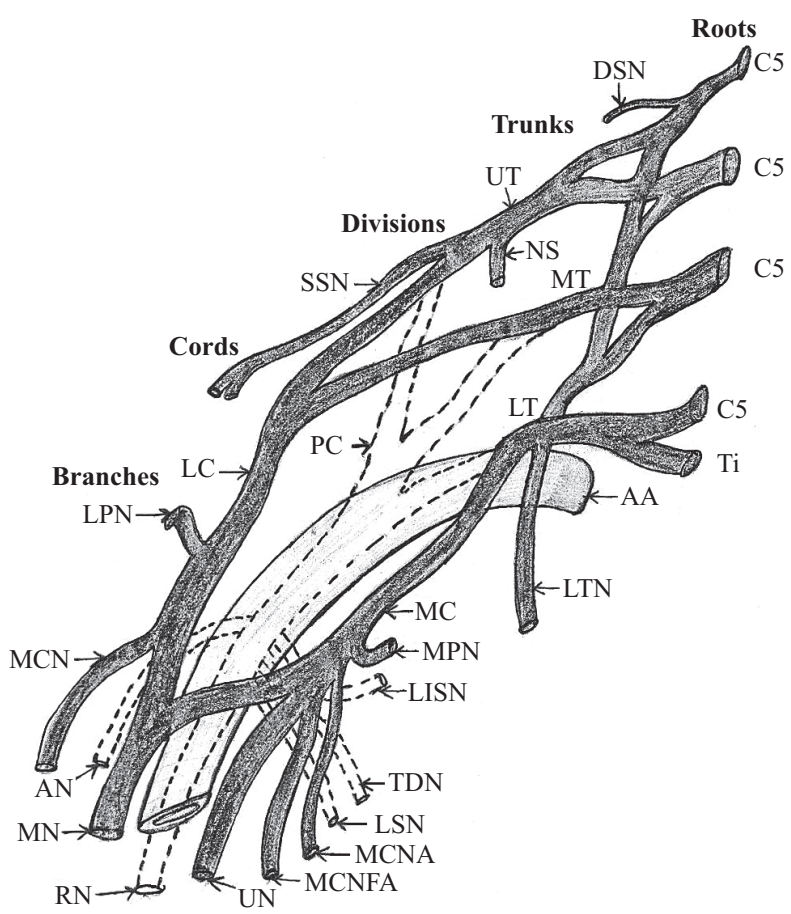

Fig. 3: Schematic drawing of the normal brachial plexus. UT - upper trunk, MT - middle trunk, LT - lower trunk, LC - lateral cord, MC - medial cord, PC - posterior cord, DSN - dorsal scapular nerve, SSN - suprascapular nerve, LPN - lateral pectoral nerve, MPN - medial pectoral nerve, $\mathrm{MCN}$ - musculocutaneous nerve, UN - ulnar nerve, $\mathrm{RN}$ - radial nerve, $\mathrm{MN}$ - median nerve, $\mathrm{AN}$ - axillary nerve, MCNA - medial cutaneous nerve of arm, MCFNA - medial cutaneous nerve of forearm, USN - upper subscapular nerve, LSN - lower subscapular nerve, TDN - thoracodorsal nerve, NS - nerve to subclavius, LTN - long thoracic nerve, AA - axillary artery of its proximity to the heart, its large contractile mass, the lack of appreciable morbidity caused by its displacement to the thorax, and the technical simplicity in transposing it to the heart. An abnormal communication between the thoracodorsal nerve and other branches of the posterior cord could possibly jeopardize this life saving maneuver.

Presumably in view of the common trunk of thoracodorsal and lower subscapular nerves encountered in the current investigation, caution should be exercised while mobilizing the latissimus dorsi into the left pleural and mediastinal cavities. Furthermore, injury to the common trunk for the origin of thoracodorsal and lower subscapular nerves would result in paralysis of latissimus dorsi and teres major. Unscrupulous mobilization can, therefore, jeopardize the common trunk, leading to unsuccessful surgical outcome in such patients.

An earlier case report described a communication between the median nerve and musculocutaneous nerve coexistent with an accessory brachial muscle. Interestingly, a twig was observed to pass from this site of communication to innervate the coracobrachialis muscle (6).

Our study carries special significance in view of neural connections observed not only between the cords, but also between the nerves of the posterior cord.

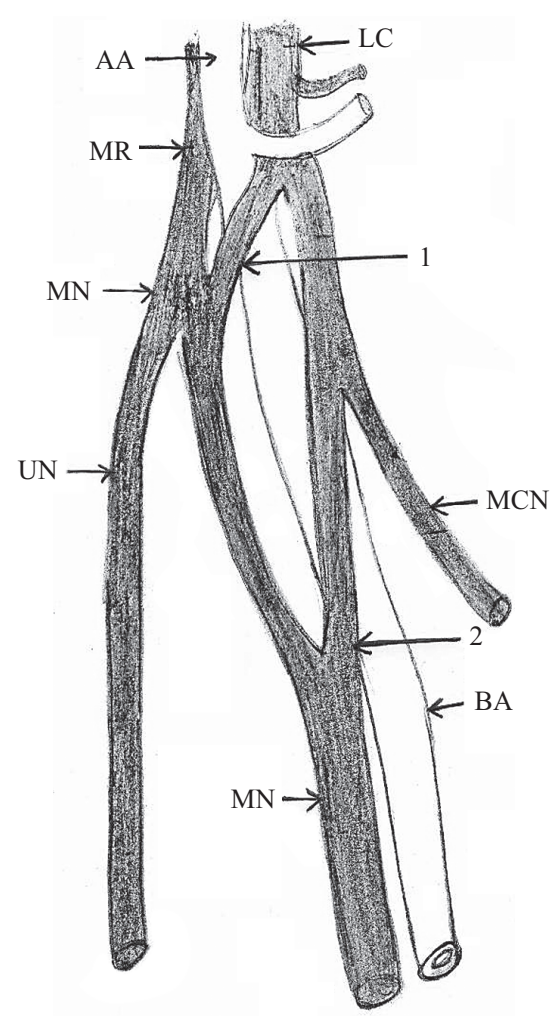

Fig. 4: Schematic drawing to show the neural communication between lateral and medial cord. MC - medial cord, $\mathrm{LR}$ - lateral root, $\mathrm{MR}$ - medial root, $\mathrm{MN}$ - median nerve, UN - ulnar nerve, AA - axillary artery, MCN - musculocutaneous nerve, BA - brachial artery, 1,2 - neural communication 


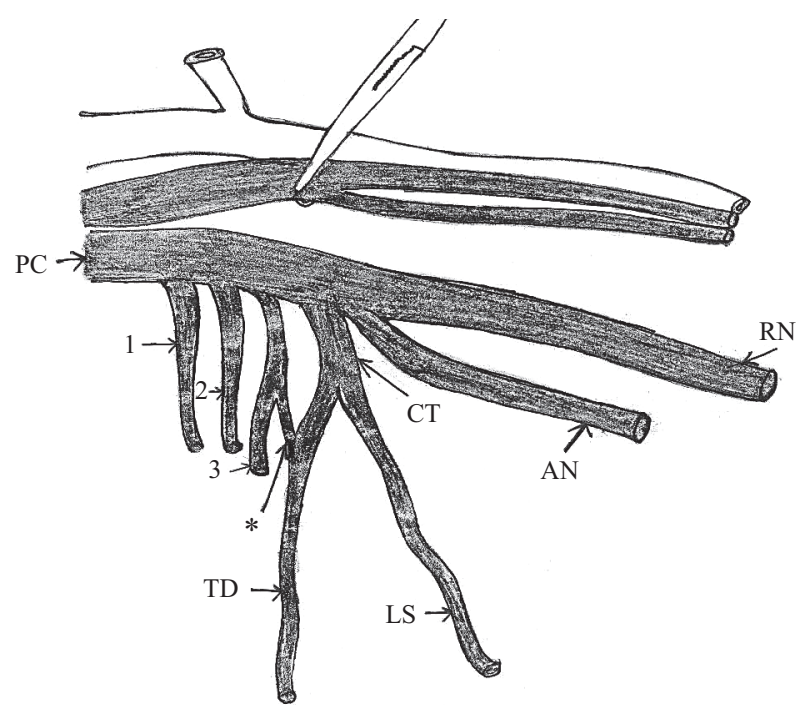

Fig. 5: Schematic drawing to show the variation in the branching pattern of the posterior cord of left side. $\mathrm{PC}$ - posterior cord, 1 - upper subscapular nerve, 2 - upper subscapular nerve, 3 - upper subscapular nerve, CT - common trunk, TD - thoracodorsal nerve, LS - lower subscapular nerve, LD - latissmus dorsi muscle, * - communicating twig of $3 \mathrm{rd}$ upper subscapular nerve to the common trunk of thoracodorsal and lower subscapular nerves, $\mathrm{RN}$ - radial nerve, $\mathrm{AN}$ - axillary nerve

A relation between the neural anomalies and variant arterial branching pattern has been stated previously, although our case report did not reveal any vascular abnormality (8).

The shoulder and axillary regions are often approached by anesthetists, orthopedic and plastic surgeons. The infraclavicular approach for the brachial plexus block is effective. It produces reliable anesthesia and is associated with minimal complications and side effects (13). It is relevant to suggest, therefore, that clinicians be conscious of the branching and topographical relations of the brachial plexus and their possible anomalies. Lack of knowledge of these neural variations may lead to inadvertent intra-operative and post-operative complications resulting in sensory, motor and trophic signs and symptoms. The knowledge of these neural variants may be employed to explicate arbitrary clinical signs and symptoms. Moreover, these anomalies may be responsible for several nerve palsies and vascular pathologies.
Acquaintance of these variations may be successfully used by clinicians while performing image processed 3-D magnetic nuclear resonance scans in which a single composite image of the brachial plexus is visualized. This could allow surgical maneuvers in the axillary region to be improvised.

\section{Conclusions}

The anatomy, topographical relationship and branching pattern of the brachial plexus cords should be the subject of much consideration when diagnosing clinical conditions, especially neuropathies. Surgeons, radiologists and anesthetists need to be careful before attempting to approach this vital region.

\section{References}

1. Alnot JY and Huten B. La systematisation du plexus brachial. Rev. Chir. Orthop. 1997; 63:27-34.

2. Arora L, Dhingra R. Absence of musculocutaneous nerve and accessory head of biceps brachii:a case report. Indian J Plast Surg 2005; 38(2):144-146.

3. Ballesteros LE, Ramirez LM. Variations of the origin of collateral branches emerging from the posterior aspect of the brachial plexus. J Brachial Plex. \& Peripher. nerve inj. 2007; 2:14.

4. Goyal N, Harjeet, Gupta M. Bilateral variant contribution sin the formation of median nerve. Surg Radiol Anat 2005; 27(6):562-565.

5. Kerr AT. The brachial plexus of nerves in man, the variation in its formation and branches. Am J Anat 1918; 23:285-395.

6. Mehta V, Yadav Y, Arora J, et al. A new variant in the brachium musculature with reinforced innervation from a median - musculocutaneous nerve communication. Morphologie 2009; 93(301):63-6.

7. Moore KL, Dalley AF. Upper Limb: In clinically oriented anatomy, pp 773-784, edited by Moore KL, Dalley AF, Philadelphia, 2006.

8. Nakatani T, Tanaka S, Mizukami S. Superficial brachial arteries observed in bilateral arms. Kaibogaku Zasshi. 1996; 71:308-12.

9. Nguyen TH, Hoang TA, Dash N, Christlieb I, Lupetin AR, Beckman I, Schapiro RL, Magovern GJ. Latissimus dorsi Cardiomyoplasty :Radiological findings. Am J Roent 1988; 150(3):545-7

10. Pais D, Casal D, Santos A and O'Neill JG. A variation in the origin of the median nerve associated with an unusual origin of the deep brachial artery. J Morphol Sci 2010; 27(1):35-8.

11. Pandey SK and Shukla VK. Anatomical variations of the cord of brachial plexus and the median nerve. Clin. Anat. 2007; 20:150-6.

12. Saddler TW. Muscular system. In Langman's Medical embryology. 10th ed. Philadelphia: Lippincott Williams and Wilkins, 2006:146-7.

13. Salazar CH, Espinosa W. Infraclavicular brachial plexus block: Variation in approach and results in 360 cases. Regional Anesthesia and Pain Medicine 1999; 24(5):411-416.

14. Senecail B, Delmas A and Quereux P. Donnees morphologiques et biometriques sur la constitution du plexus brachial de l'homme. Bull. Assoc. Anat. 1979; 63:513-25.

15. Tubbs RS, Loukas M, Shahid K, Judge T, Pinyard J, Shoja MM, Salppey JB, McEvoy WC, Oakes WJ. Anatomy and quantitation of the subscapular nerves. Clin anat 2007; 20:656-659.

16. Uzun A, Bilgic S. Some variations in the formation of the brachial plexus in infants. Tr J of Med Sci 1999; 29:573-7.

17. Venieratos D. and Anagnostopoulous S. Classification of communications between the musculocutaneous and median nerves. Clin. Anat. 1998; 11:327-31

18. Walsh JF. The anatomy of the brachial plexus. Am J Med Sci 1877; 74:387-428.

Received: 14/03/2011

Accepted in revised form: 02/08/2011

\section{Corresponding author:}

Dr.Vandana Mehta, M.S., Associate professor (Anatomy), VMMC \& Safdarjung Hospital, New Delhi, India; e-mail: drvandanamehta@gmail.com 